\title{
Modelling LI grammatical attrition through language acquisition: a reply to comments
}

\section{Glyn Hicks and Laura Domínguez}

University of Southampton, UK

We wish to begin by thanking the Second Language Research editors for the opportunity to present our model of L1 grammatical attrition ('Attrition via Acquisition', AvA) as a keynote article. We also warmly thank the colleagues who have prepared commentaries that shed further light on the challenges that lie ahead for our model, and indeed for the field of L1 grammatical attrition. Here we offer responses, observations and clarifications to the main points raised in their commentaries.

Within the broad and rapidly developing field of language attrition research, we reiterate at the outset that our own principal interest lies in the specific phenomenon that we refer to as L1 grammatical attrition. This specific focus on attrition within the grammar itself is significant, as it is distinct from other changes to L1 processing, cognition or performance that may be attested for bilinguals. Our keynote article (Hicks and Domínguez 2019) seeks to emphasise that the nature of grammatical attrition may have quite fundamental implications for current models of the human language faculty and its development during the lifespan of an individual. It is welcoming to receive wholehearted agreement on this point from a number of the commentaries (Lohndal, Putnam, Sorace, and Westergaard). The AvA model that we present in the keynote article aims to provide a platform for future research that will elucidate and account for the processes by which substantive changes to specifically grammatical representations arise within a mature L1 grammar. AvA is designed to satisfy a number of key criteria that we identified for a model of grammatical attrition, which we take in turn below.

\section{A model that allows grammatical attrition}

Firstly, the model should permit L1 grammatical attrition - at least in principle, and to some degree. Here we will restate our definition of grammatical attrition as a "potentially enduring modification to morphosyntactic properties of an end-state grammar under linguistic 
pressure" (Hicks and Domínguez: 2019: xxx). While grammatical attrition has a possible role in heritage language acquisition (as highlighted in the commentaries of Westergaard, Montrul, Putnam, and Perpiñán), our focus in the keynote article is only on changes to "end-state" grammars, ${ }^{1}$ as emphasised specifically by Sorace. We are certainly encouraged by support from commentaries such as Montrul's, indicating that attrition that consists of the 'addition' of L2-based grammatical options offers a promising explanation for the properties of heritage grammars. ${ }^{2}$ Yet equally, other commentaries present potential counter-examples to predictions of AvA based on attrition in heritage grammars rather than end-state grammars: Putnam indicates that AvA does not account for known morphosyntactic patterns in attrition with examples from heritage grammars; Westergaard and Perpiñán provide possible counter-evidence from heritage language acquisition to the prediction of AvA that L2 acquisition of the relevant property must have taken place for attrition to occur. ${ }^{3}$ Through formalising grammatical attrition as a specific subcategory of bilingual acquisition, the AvA model's broader - and most ambitious - aim is to provide a framework for capturing a range of patterns in bi/multilingualism acquisition within mainstream theoretical models of the language faculty and language acquisition: Montrul, for example, illustrates that the model may provide a framework for understanding other linguistic properties of bilinguals, such as fossilization in L2 grammars. While we maintain that, contra Gürel, a model of language acquisition can in principle accommodate both heritage language acquisition and (adult) grammatical attrition, properly addressing the possibility of attrition within heritage language acquisition is not our principal aim in the keynote article: this will require further articulation of AvA to take into account the influence of the particular environmental, processing and grammar-internal circumstances of heritage language acquisition. While the same mechanisms of attrition may be available, the conditions and characteristics will necessarily differ. As Montrul highlights, for example, further articulation of AvA is required to properly account for the influence of maturational or age-related factors in the susceptibility of L1 grammars to change. This task remains for future research.

Exactly what extent of change to end-state grammars is possible, in what grammatical properties, through which grammatical mechanisms, and under what environmental conditions remain the key empirical questions for this field (see Schmid's commentary in this regard). We have a long way to go in working towards responses to these questions that we can be confident about; Schmid (2019: xxx) also draws attention to the "relatively limited experimental evidence available in the field so far." As mentioned, our starting point is that changes to the mature L1 grammars are, in principle, possible. As emphasised by Stringer, for example, even this is not an uncontroversial position, particularly given the traditional "deficit view of attrition" (Montrul 2019: xxx) as the loss or erosion of some property. ${ }^{4}$ Within a formally (grammatically) explicit approach to attrition, which our model seeks to facilitate, terms such as "loss" or "erosion" lack clear import as far as grammatical 
representations are concerned. As such, as noted above, we adopt a broader definition of grammatical attrition as a type of modification to morphosyntactic properties of an end-state grammar. The nature of the possible grammatical modifications and the mechanisms that give rise to them are separate, empirical matters. Under this definition, grammatical attrition could in principle either consist of some kind of "loss" of L1 representations and/or a different kind of change. We present arguments that modification is in fact likely either to supplement existing L1 feature specifications with additional forms based on a corresponding L2 representation or to involve fluctuation between L1 and L2-based grammatical options; a possible extension, for example, might involve whether other sorts of modification are also available, for example an 'overwriting' of L1 forms with the L2 equivalents. Evidently, it remains to fully uncover the nature of the mechanisms that lead to grammatical change, yet contra Stringer, we argue that from a formal perspective it makes no sense to distinguish one type of modification to the grammar as constituting attrition from another that does not. ${ }^{5}$

Sorace, on the other hand, proposes that even understood simply as change to an L1 grammar, attrition does not obtain within the grammar itself: "mental representations of grammatical knowledge in mature L1 speakers are NOT affected" (Sorace 2019: xxx). By the same token, Genevska-Hanke highlights that the relative rarity of attrition has been taken as evidence that the locus of attrition is linguistic behaviour, rather than knowledge. Sorace's argument, following Chamorro, Sorace \& Sturt (2016), is based on the evidence that L1 attrition may be reversed in the case of re-exposure. Noting that the commentaries by Gürel, Genevska-Hanke and Schmid also emphasise recent research on the reversibility of attrition, we do not make any specific claims in the keynote article (or indeed here) about reversibility and we do not accept Sorace's premise that a prerequisite for grammatical attrition is that it must be irreversible. ${ }^{6}$ Moreover, the criticism that we "try to account for everything in formal grammatical terms when there are plausible non-grammatical explanations" (Sorace 2019: $\mathrm{xxx}$ ) is, we believe, misplaced: we are only proposing grammatical explanations for linguistic phenomena that exhibit grammar-internal interactions, typically between syntactic distribution, morphosyntactic realisation and semantic interpretation. We take the fact that the commentaries by Lohndal, Putnam and Westergaard advance particular theoretical approaches to the grammatical component in ways they claim to be appropriate to understanding attrition as evidence of their acceptance of our assumption that the grammar itself is a possible locus of L1 attrition, contra Sorace.

\section{A model that incorporates a formally explicit generative grammar}

Evidently, making progress in understanding what grammatical changes are attested in L1 attrition requires a formally explicit, precise and theoretically informed characterisation of the 
relevant grammatical properties of L1 and L2 grammars, as has become standard practice in modern generative research into first and second language acquisition, for example. A further aim of the model, then, is to incorporate a grammatical component whose properties can be articulated in formally explicit, theoretically informed, linguistic terms: where the relationship between the relevant grammatical properties of the L1 and L2 are known to be critical to the likelihood of L1 attrition, it is essential that we can articulate these differences. Moreover, given that grammatical attrition, where attested, appears to be typically borne out in small adjustments to specific L1 grammatical properties (rather than wholesale reorganising of the grammar), it is essential that the model of the grammatical component can analyse such fine-grained variation.

To this end we chose to adopt a feature-based model which is essentially 'mainstream' in that it is theoretically well established and has already demonstrated potential for implementation within language acquisition research due to the Feature Reassembly Hypothesis (Lardiere 2008, 2009). However, as highlighted in the commentaries by Lohndal and Putnam, alternative models of the grammatical component may be particularly amenable to being incorporated into an attrition model. We fully acknowledge Lohndal and Putnam's claims that an exponency-based model might in principle allow for different understandings of why particular modules or interfaces within the grammar might be more or less susceptible to attrition: it is our explicit intention that the model of attrition must be informed by considerations of grammatical architecture in linguistic theory. Moreover, as Westergaard and Lohndahl emphasise, the model of attrition may also allow current understandings of bilingual/multilingual data to inform formal grammatical models (Lohndahl) and the cognitive architecture of the language faculty (Westergaard). We leave it for future research to assess the validity of these changes to specific components of the overall model.

\section{A model that accommodates the known patterns in grammatical attrition}

Gürel, Perpiñán, Putnam, Lohndal, Iverson and Westergaard probe the predictive capabilities of the model in its present state. Putnam and Lohndal emphasise that the model needs to be articulated further in order to add predictive power. This in fact reflects the intentional design of the attrition model, and in this respect the acquisition model of Lidz and Gagliardi (2015) on which AvA is based is the same. Our aim is to construct a model in which all of the relevant considerations may in principle have the kind of influence on the likelihood of grammatical attrition that we are just beginning to understand they have, with the restrictions on this to be set be the mechanisms of the individual components. This is recognised by Schmid (2019: xxx), who judges that the AvA model "has the capacity of generating testable hypotheses and workable research questions that can advance our 
understanding of the attritional process". Necessarily at this stage in the development of the model, not all of these components are fully specified. For example, as mentioned above, Montrul highlights the need for AvA to account for the influence of maturational or agerelated factors in the susceptibility of L1 grammars to change. There are various possibilities within the model: it is an open empirical matter whether, in what ways, and in which combination such factors influence parsing, perceptual intake, access to UG, and inferential intake. $^{7}$ As another example, concerning the nature of the grammatical component specifically, we agree with Lohndal (2019: xxx) that "By articulating an even more detailed formal implementation, the model would be able to move closer to making genuine predictions about cross-linguistic patterns in attrition." This would entail, for a given theoretical account of the grammatical component, a formal comparative analysis of the relevant morphosyntactic properties of the target 'attriting' L1 property and any relevant corresponding properties of the L2. Indeed, this is the approach we attempted to take in our own earlier work on null and postverbal subjects in Spanish bidialectals (Domínguez \& Hicks, 2016). Any predictions arising from the AvA model will be constrained by the availability of intake and how easily reassembly can take place; these two issues are mediated by the similarities and differences between structures in the L1 and L2. In summary, once we know the language pairing and have a formal analysis of the target structures in both languages couched within a given theory of the grammatical component, the model will generate testable predictions for that particular context. ${ }^{8}$

Moreover, while the model does not predict a priori whether any given grammatical property will be attrited, Gürel and Westergaard identify a prediction that attrition of an L1 grammatical property should entail the acquisition of that property in the L2. ${ }^{9}$ A recent case study by Baker (2019) of an attriting L1 German speaker is the first empirical work we know of that explicitly seeks to test the AvA model and focuses specifically on this prediction. As an illustration of how the model can be applied to make predictions for grammatical attrition, Baker studies an L1 German speaker resident in the UK for 19 consecutive years. He tests the speaker's knowledge of anaphoric binding of reflexive pronouns (a grammatical property known to be highly sensitive to syntactic conditions) in two syntactic environments where German contrasts with English:

1) a. You give Ullrich $_{i}[\mathrm{NP}$ a photo of himself $i$ ]

b. $\quad *$ Du gibst Ullrich $\left[\right.$ [NP ein Foto von $\left.\operatorname{sich}_{\mathrm{i}}\right]$

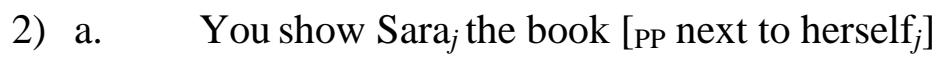

b. *Du zeigst Sara ${ }_{j}$ das Buch [PP neben $\left.\operatorname{sich}_{j}\right]$

In English, reflexives inside a "picture-NP" (1a) and locative PP (2a) can take an object antecedent, while both equivalents are ungrammatical in German (both languages permit a 
subject antecedent). For the PP conditions, Baker's case study participant patterns with the German control group in rating the German structure (e.g. (2b)) highly unacceptable in an Acceptability Judgment Task. There is thus no attrition, and for the English version of the task, (e.g. (2a)) she also diverges from the native English control group, considering it unacceptable. On the other hand, in the picture-NP conditions, the case study participant shows a marked divergence from the German control group, rating the German structure (e.g. (1b)) more acceptable than the native German controls. Crucially, this L1 attrition is reflected in her performance on the English version of the task (e.g. (1a)), converging in this case with the native English control group. In summary, Baker's results were consistent with AvA's prediction that attrition of an L1 grammatical property should entail the acquisition of that property in the L2. L1 attrition is not attested for the binding property that was not acquired in the L2 (binding of a reflexive in a locative PP by an object), whereas attrition is attested for the binding property that is demonstrably acquired in the L2 (binding of a reflexive in a picture-NP by an object).

\section{A model that resolves the paradox in modelling grammatical attrition}

The AvA model addresses what we refer to as a 'paradox' facing any formal model of grammatical attrition, namely that the broad empirical pattern that it must explain is such that while changes to a fully acquired mature L1 grammar (even acquired under monolingual conditions, i.e. outside of the complicating considerations of heritage language acquisition) are possible, the phenomenon itself is known to be highly constrained. Gürel (2019: xxx) responds that "the fact that modification to the well-developed L1 system is constrained and selective is not a paradox", yet this is a misunderstanding of our view. The paradox concerns not the fact that grammatical attrition exists or is selective, but how to account theoretically for this fact. For example, on the one hand, if L1 attrition of end-state grammars is not - even in principle - an option that the language faculty provides, then how can the restricted set of cases where it occurs be accommodated? On the other hand, if such L1 grammatical attrition is a possibility that the language faculty provides, then why is it not more widely attested once contact with a second language starts in adulthood? Our approach to resolving that paradox is to assume the possibility of L1 grammatical attrition, with the constraints on grammatical attrition determined by the specific combination of grammatical and processing mechanisms that are required to engender change in an end-state L1 grammar.

An alternative resolution of the attrition paradox, raised by Iverson, is that its premise is essentially incorrect, and that L1 grammatical attrition is not in fact as rare as we have come to assume; it just takes different forms. Iverson mentions a number of examples of minor dialectal/stylistic variants in English (e.g. preposition stranding versus pied-piping, anymore without the standardly required NPI licensor) and asks what the difference is between these 
properties being added to an end-state grammar and grammatical attrition, under the AvA model. The individual properties Iverson mentions probably merit different kinds of responses (for some, we are not convinced that the properties will not already be present in the child L1 input, for example) yet in principle the question is valid. While this is not traditionally considered as attrition, in terms of the grammatical mechanisms involved it is possible that there is no formal difference between such adjustments to the L1 and grammatical attrition; each concerns the addition of properties to the L1 grammar due to a revised input. However, it does seem that the possible modifications to the L1 grammar represent a scale depending on how deeply acquiring the new variant properties influences the existing grammar, and this probably determines the nature of the input and intake required to make that modification: we would need to examine each one case-by-case based on the nature of the grammatical differences (e.g. feature specifications) between the L1 and the 'new' grammatical variant to be acquired.

Evidently, theoretical approach to attrition that we take in Hicks and Domínguez (2019) is relevant to advancing our understanding of the phenomenon itself, and many questions remain open. But it is also relevant to understanding the nature of language acquisition. The AvA model is described in terms of clarifying the procedures giving rise to grammatical attrition, yet it is designed as a revision to an L1 acquisition model (Lidz \& Gagliardi, 2015) ultimately in order to unify all types of language acquisition, under varied input conditions. L1 grammatical attrition is simply a possible outcome of acquisition under a particular set of external conditions (linguistic environment, and changes to it over time) and internal conditions (dependent upon the states of a speaker's existing grammars, and for which languages). In other words, there is nothing that is unique to grammatical attrition: as noted by Belletti and Iverson, a welcome innovation of our model is that the mechanism of attrition exists independently of the language faculty via the language acquisition device. In other words, there is nothing 'special' about the process: as Iverson (2019: xxx) highlights, "phenomena such as attrition, even if ecologically rare, are not unexpected". This is a nice characterisation of the way we have tried to the formalise attrition and resolve the attrition paradox.

\section{Summary}

In summary, we are delighted to have received such varied and insightful commentaries that engage robustly with the assumptions, theoretical devices, and predictions of the AvA model as presented in Hicks and Domínguez (2019). Within the limited scope of this short response, we have tried to engage with some of the most salient comments and themes arising from these commentaries. It is not easy to draw out points that are common to all of them. 
However it appears that one of the least controversial assumptions we made is that L1 grammatical attrition is a phenomenon that has the potential to advance our current understanding of the language faculty architecture. Given this, it is surprising that a plausible model of attrition within generative linguistic theory has, to date, been conspicuously lacking. AvA seeks to provide such a model, and as summarised above, its specific articulation responds to a number of desiderata that we identified for any generative model of attrition. Strikingly few of the comments in the commentaries either suggested that such a model was not required or raised objections to these criteria which guided the articulation of the model. Many of the principal features of AvA were quite widely praised in the commentaries, although with some exceptions: we acknowledge that not all of the commentaries agree that grammatical attrition is indeed a possible process of the language faculty. We are pleased to receive support for the incorporation of a formally explicit generative grammar into an attrition model, for the integration of attrition with existing acquisition mechanisms, and for the understanding of grammatical attrition as a process not principally of loss, but of adding grammatical options to the existing L1 set.

We developed the AvA model to provide a platform for further generative research into grammatical attrition. As a refinement of Lidz \& Gagliardi's (2015) L1 acquisition model, it establishes a critically important role for attrition and bilingual/multilingual acquisition in providing insights into our understanding of language acquisition and the language faculty. Empirical studies are urgently required in order to better understand the possible extent of grammatical attrition and the linguistic and extralinguistic conditions that favour it, for a range of grammatical properties across a range of L1/L2 language pairings. We hope that AvA provides a platform for such studies, through which the model's predictions can be tested and its articulation further refined.

\section{Declaration of Conflicting Interest}

The authors declare that there is no conflict of interest.

\section{Funding}

The author(s) received no financial support for the research, authorship, and/or publication of this article.

\section{ORCID iD}

Glyn Hicks https://orcid.org/0000-0003-4126-8655

Laura Domínguez https://orcid.org/0000-0003-2701-2469

\section{References}


Baker, L. (2019). Grammatical Attrition in L1 German: Investigating the Attrition via Acquisition Model. MSc dissertation, University of Southampton.

Belletti, A. (2019). Attrition in acquisition with principles and parameters. Second Language Research. Epub ahead of print.

Chamorro, G., Sorace, A., \& Sturt, P. (2016). What is the source of L1 attrition? The effect of recent L1 re-exposure on Spanish speakers under L1 attrition. Bilingualism: Language and Cognition, 19(3), 520-532. doi:10.1017/S1366728915000152

Domínguez L. and Hicks G. (2016) Synchronic change in a multidialectal community: Evidence from Spanish null and postverbal subjects. In: Cuza A., Czerwionka L., and Olson D. (eds), Inquiries in Hispanic linguistics: From theory to empirical evidence. Amsterdam: John Benjamins, pp. 53-72.

Genevska-Hanke, D. (2019). Some Notes on the Nature of L1-Attrition and Its Modeling. Second Language Research. Epub ahead of print.

Gürel, A. (2019). Is there a paradox in adult L1 grammatical attrition? Second Language Research. Epub ahead of print.

Hicks, G. \& Domínguez, L. (2019) A model for L1 grammatical attrition. Second Language Research. Epub ahead of print. Published online: 30 July 2019 (doi: 10.1177/0267658319862011).

Iverson, M. (2012). Advanced language attrition of Spanish in contact with Brazilian Portuguese. Unpublished doctoral dissertation, University of Iowa.

Iverson, M. (2019). Unbridled attrition? Second Language Research. Epub ahead of print.

Lardiere, D. (2008). Feature assembly in second language acquisition. In J. M. Liceras, H. Zobl, \& H. Goodluck, (Eds.), The role of formal features in second language acquisition, pages 106-140. New York: Lawrence Erlbaum Associates.

Lardiere, D. (2009). Some thoughts on the contrastive analysis of features in second language acquisition. Second Language Research 25, 173-227.

Lidz, J. \& Gagliardi, A. (2015) How Nature Meets Nurture: Universal Grammar and Statistical Learning. Annual Review of Linguistics 1(1), 333-352.

Lohndal, T. (2019). A distributed architecture of L1 attrition. Second Language Research. Epub ahead of print. 
Montrul, S. (2019). Attrition, addition, and age. Second Language Research. Epub ahead of print.

Montrul S., Bhatt R., \& Girju, R. (2015) Differential object marking in Spanish, Hindi and Romanian as heritage languages. Language 91: 564-610.

Perpiñán, S. (2019). What can the 'attrition via acquisition' model predict? Second Language Research. Epub ahead of print.

Putnam, M. T. (2019). Lexicalizing exponents in multilingual grammars, Second Language Research. Epub ahead of print.

Schmid, M. S. (2019). On attrition, spaceships and viruses. Second Language Research. Epub ahead of print.

Sorace, A. (2011). Pinning down the concept of "interface" in bilingualism. Linguistic Approaches to Bilingualism, 1, 1-33.

Sorace, A. (2019). L1 attrition in a wider perspective. Second Language Research. Epub ahead of print.

Stringer, D. (2019). Not all acquisition entails attrition: The persistence of syntax in the teeth of lexical breakdown. Second Language Research. Epub ahead of print.

Westergaard, M. (2019). Attrition via acquisition: The importance of development in small steps: A Commentary on 'A model for L1 grammatical attrition' by Glyn Hicks and Laura Domínguez. Second Language Research. Epub ahead of print.

1 The term "end-state grammar" is retained here for familiarity, but note that the term itself should be revisited in light of the assumption that changes to such grammars are possible.

2 Although Montrul also indicates that the relevant properties of grammatical attrition consistent with the AvA model were also found in the grammars of adult L2-speaking migrants (Montrul et al. 2015).

3 On this specific prediction of AvA, see Section 3 below.

4 Specifically, Stringer notes of our analysis of the attrition data in bidialectals (Domínguez \& Hicks 2016) that "speakers may add dialectal variations without losing their L1 possibilities. On a narrower definition, such cases do not involve language attrition." (Stringer 2019: xxx) 
5 Evidently, the current state of understanding in grammatical attrition raises a central question for the field concerning the term "attrition" itself, originally associated with 'loss' and 'erosion', as may be the case with attrition of broader linguistic abilities.

6 Recall here our definition of grammatical attrition noted above based on "potentially enduring modification to morphosyntactic properties" (Hicks and Domínguez: 2019: xxx), or equally Gürel and Y1lmaz's (2011: 222) definition as “an unconscious rearrangement or restructuring of the L1 grammar due to L2 contact, but not as a drastic loss/decay as in the case of pathological conditions." Under the AvA model, changes in the input may lead to change in the grammar, hence if the input conditions are reversed then it is expected that further changes in the grammar may take place, potentially including what we know as reversibility.

7 A similar observation about the currently underspecified mechanisms of some of the modular components is made by Gürel and Schmid.

8 It is also worth considering the predictive power of the available alternatives in this respect, principally the current version of the Interface Hypothesis. Unlike the comparatively clear distinctions and predictions of the original Interface Hypothesis as applied to attrition (and tested, most notably, by Iverson 2012) Sorace's (2019: xxx) commentary makes the case for "a continuum of conditions on syntactic realization, ranging from more 'internal' to more 'external' and involving different types of cognitive processes". The predictions of that approach are obscure at best.

9 As noted above, Westergaard provides a counter-example to this prediction, though it is based on heritage language acquisition rather than adult L1 grammatical attrition. 